\title{
Erroneously elevated immature reticulocyte counts in leukemic patients determined using a Sysmex XE-2100 hematology analyzer
}

\author{
Jungwon Huh • Heewon Moon • Whasoon Chung
}

Received: 11 January 2007 / Accepted: 7 May 2007 /Published online: 25 May 2007

(C) Springer-Verlag 2007

\begin{abstract}
The immature reticulocyte fraction (IRF) in peripheral blood, as determined by automated reticulocyte analysis, is calculated using the sum of medium and highly fluorescent reticulocyte numbers and provides information about erythropoietic activity in bone marrow. The purpose of this study was to investigate erroneously elevated IRF in leukemic patients, as determined using a Sysmex XE-2100 hematology analyzer (Sysmex, Kobe, Japan). Normal reticulocyte scattergram patterns show regions corresponding to reticulocytes located between matured RBCs and an upper particle (UPP) region, which show a continuum of nonseparated fraction. The UPP represents erythroblasts and some immature reticulocytes. As a control group, peripheral blood was taken from patients with benign hematologic diseases, and their reticulocyte scattergrams all showed a normal pattern; UPP values were all less than 100 . However, the reticulocyte scattergrams of 5 of 11 leukemia patients showed abnormal patterns and displayed a gap between RBC and reticulocyte regions. Three patients showed a flag with a message such as "RET Abn Scattergram". IRF results were elevated in these five patients, and their UPP values were above 100 . For the remaining six leukemia patients with a normal reticulocyte scattergram pattern, immature reticulocytes were not markedly increased, and UPP values were less
\end{abstract}

\footnotetext{
J. Huh $\cdot$ H. Moon $\cdot$ W. Chung

Department of Laboratory Medicine, Ewha Womans University

College of Medicine,

Seoul, South Korea

J. Huh $(\square)$

Mokdong Hospital, Ewha Womans University

College of Medicine,

Mokdong 911-1, Yangcheon-Gu,

Seoul 158-710, South Korea

e-mail: JungWonH@ewha.ac.kr
}

than 100 . The findings of the present study demonstrate that IRF results may be erroneously elevated in leukemia patients and indicate that hematologists should examine reticulocyte scattergrams and UPP values carefully.

Keywords Immature reticulocyte - Leukemia . Sysmex XE-2100 · Reticulocyte scattergram · UPP

\section{Introduction}

Reticulocyte counts provide useful information about bone marrow erythropoietic activity, the rate of reticulocyte delivery from bone marrow into peripheral blood, and the rate of reticulocyte maturation [1].

Automated reticulocyte enumeration based on flow cytometry is rapid and objective and requires less technical labor than manual slide-based techniques. Furthermore, automated reticulocyte enumeration also provides accurate information on the age distribution of the reticulocyte population. The reticulocyte population is divided into fractions, which show low, medium, or high RNA fluorescence intensities. Young and immature reticulocytes are highly fluorescent due to their high RNA contents, whereas old and maturing reticulocytes show low levels of fluorescence.

The immature reticulocyte fraction (IRF) represents the sum of medium and highly fluorescent reticulocytes. Moreover, an elevation in IRF may be the first sign of hematologic recovery in the majority of patients receiving chemotherapy or of engraftment in those that have undergone bone marrow transplantation [2-4].

As the fluorescence intensities of reticulocytes form a continuum, accurate analysis of fluorescence scattergrams is critical for reticulocyte enumeration. However, the 
fluorescence of reticulocytes may be affected by interferences from cellular elements, cellular inclusions, RBC autofluorescence, and others [1].

Interestingly, we discovered a small number of leukemia cases with markedly elevated IRF values. Accordingly, we undertook this study to investigate erroneously elevated IRF in leukemia patients using a Sysmex XE-2100 hematology analyzer (Sysmex, Kobe, Japan).

\section{Materials and method}

This study group was composed of 11 patients diagnosed as having leukemia between January 2005 and August 2006. The control group included 39 peripheral blood specimens from patients with benign hematologic diseases. Their nucleated RBC counts per $100 \mathrm{WBC}$ were from 0 to 15 , total reticulocytes from 0.5 to $9.6 \%$, and immature reticulocytes from 4.2 to $50.6 \%$.

For reticulocyte analysis of peripheral blood, we used a Sysmex XE-2100 hematology analyzer (Sysmex, Kobe, Japan) using a flow cytometry method utilizing a semiconductor laser. The normal reference range of our laboratory was as follows: total reticulocyte count from 0.6 to $2.4 \%$ and IRF from 1.5 to $11.3 \%$.

In reticulocyte scattergrams, the $x$-axis represents the intensity of lateral fluorescent light and the $y$-axis the intensity of the forward scattered light (Fig. 1a). The differentiation of reticulocytes in mature red blood cell is based on RNA levels because RNA is lost during differentiation into mature RBCs. Therefore, reticulocytes are identified and enumerated within a gated red blood cell population based on fluorescence intensity.

\section{a}
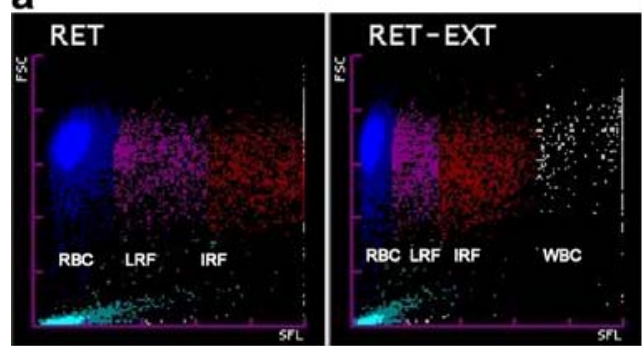

c
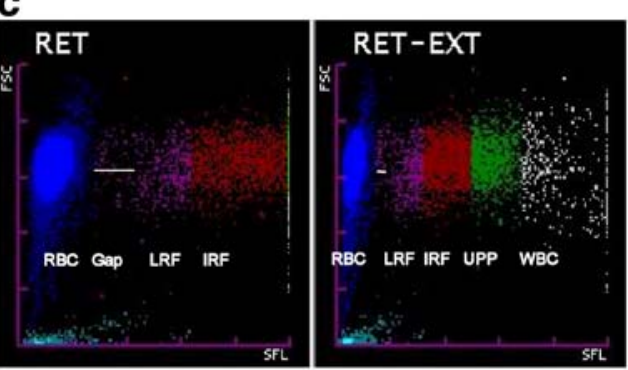

e
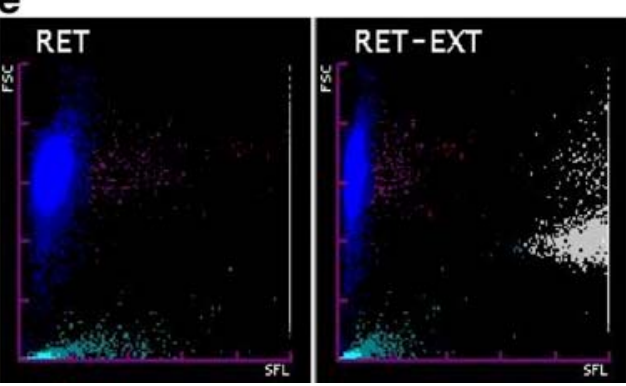

Fig. 1 Reticulocyte scattergram generated by the Sysmex XE-2100 hematology analyzer. Regions: blue mature red blood cells, pink low fluorescent reticulocyte, red immature reticulocyte, green UPP (upper particle portion), white leukocytes. a Normal control specimen pattern showing continuous distribution from RBC to IRF regions without a gap (total reticulocyte $19.4 \%$, immature reticulocyte $54.5 \%$ ). b Analyzed control specimen data with a UPP value of 0 and a WBC area value of 315. c An AML patient (case no.2) showing an abnormal

\section{b}

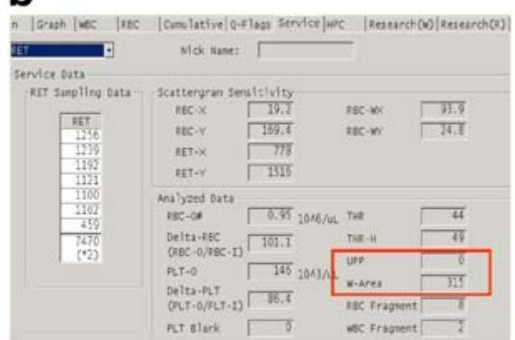

d
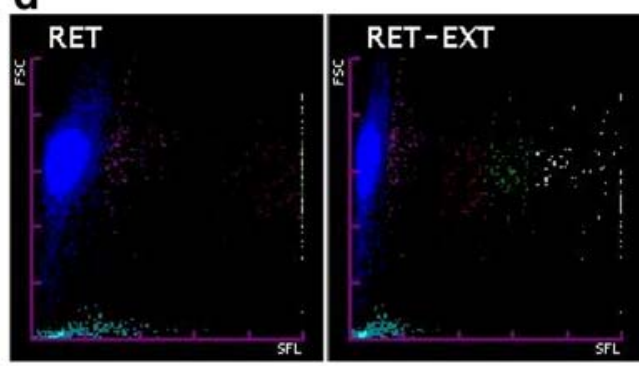

f
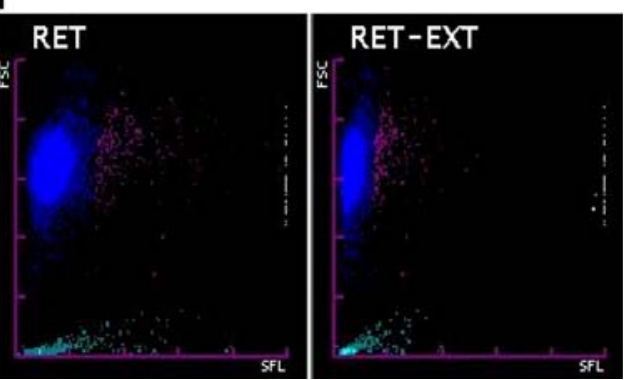

pattern with a gap between $\mathrm{RBC}$ and reticulocyte regions and many dots in the UPP region despite presence of a few NRBCs. d After leukapheresis WBC counts decreased, the gap disappeared, and dots in the UPP region reduced. e A CLL patient (case no. 11) showing a normal pattern and many dots in the WBC region due to leukocytosis, but no dots in the UPP region. $f$ An AML patient (case no. 8) showing a normal pattern with a continuous distribution between RBC and reticulocyte regions and no dots in the UPP region 
A normal reticulocyte scattergram pattern is composed of a region of reticulocytes located between regions of mature RBCs and an upper particle (UPP) region. Moreover, these fractions form a continuous non-separated distribution (Fig. 1a). Thus, UPP values indicate primarily erythroblast numbers and include some immature reticulocytes (Fig. 1b).

The dye used for reticulocyte analysis stains leukocytes, but leukocytes are not usually shown in reticulocyte scattergrams because of their high fluorescence. In extended reticulocyte scattergrams, the leukocyte region is just to the right of the UPP region, and the WBC region indicates the number of leukocytes (Fig. 1a, b).

\section{Results}

The reticulocyte scattergrams obtained from the control samples all showed a normal pattern with UPP values from 0 to 21 . However, the reticulocyte scattergrams of 5 of the 11 leukemia patients (Table 1, case nos. 1-5) showed an abnormal pattern, i.e., a clear gap was present between the $\mathrm{RBC}$ and reticulocyte regions. Moreover, the reticulocyte region was separated from the RBC region (Fig. 1c-e), whereas normal scattergrams showed a reticulocyte region located just to the right of the $\mathrm{RBC}$ region without a gap (Fig. 1a). Three patients (Table 1, case nos. 1, 2, 4) showed the flag with the message of "RET Abn Scattergram". Immature reticulocyte levels were all elevated in these five patients (Table 1, case nos. 1-5), and their UPP values were above 100. In particular, three of these five patients showed a spurious IRF (case nos. 1, 2, 5) increases. In one patient (case no. 2-1), reticulocyte counts determined by the automated and manual methylene blue stain methods differed ( 7.4 vs $2 \%$, respectively). IRF and UPP values were reduced with decrease of WBC counts in three patients (case nos. 2-4) who received therapeutic leukapheresis.

Among the six leukemia patients with normal reticulocyte scattergram patterns (cases no. 6-11), immature reticulocytes were not markedly increased, and UPP values were less than 100. One patient with CLL (case no. 11) showed a markedly elevated total WBC count and a low UPP.

\section{Discussion}

We found that IRF values were markedly elevated in some leukemia patients with abnormal reticulocyte scattergrams. These elevated IRF values could suggest increased erythropoietic activity of bone marrow. However, we consider that increased IRF in leukemic patients is not a true result because increased hematopoiesis in leukemic patients is unlikely [5].

Fluorescence scattergrams of reticulocytes may be affected by interferences such as cellular elements, cellular inclusions, RBC autofluorescence, and others [1]. One author reported that reticulocyte counting by flow cytometry might be influenced by white cells, which may be erroneously identified as reticulocytes, and demonstrated a positive correlation between highly fluorescent reticulocytes and leukocytosis [6].

However, our results demonstrate that patients with leukocytosis do not always show an erroneously increased IRF value, although IRF values tend to be elevated in patients with leukocytosis. For two patients with leukocy-

Table 1 Data of reticulocyte analysis in leukemic patients

\begin{tabular}{|c|c|c|c|c|c|c|c|c|c|c|c|}
\hline $\begin{array}{l}\text { Case } \\
\text { no. }\end{array}$ & $\begin{array}{l}\text { Age/ } \\
\operatorname{sex}\end{array}$ & Dx & $\begin{array}{l}\text { TR } \\
(\%)\end{array}$ & $\begin{array}{l}\text { IRF } \\
(\%)\end{array}$ & $\begin{array}{l}\text { Reticulocyte } \\
\text { scattergram }\end{array}$ & Flag $^{\mathrm{a}}$ & $\begin{array}{l}\text { UPP } \\
\text { value }\end{array}$ & $\begin{array}{l}\text { NRBC (/100 } \\
\text { WBC) }\end{array}$ & $\begin{array}{l}\text { WBC-area } \\
\text { value }\end{array}$ & $\begin{array}{l}\text { WBC } \\
\left(\times 10^{9} / 1\right)\end{array}$ & $\begin{array}{l}\text { Leukemic } \\
\text { cells (\%) }\end{array}$ \\
\hline 1 & $9 / \mathrm{M}$ & AML & 0.9 & 82.3 & Abnormal & + & 810 & 0 & 1,739 & 268 & 95 \\
\hline $2-1$ & $59 / \mathrm{M}$ & AML & 7.4 & 78.0 & Abnormal & + & 713 & 2 & 295 & 174 & 100 \\
\hline $2-2^{b}$ & & & 0.4 & 35.6 & Abnormal & - & 46 & 0 & 54 & 12 & $\mathrm{nc}$ \\
\hline $3-1$ & $33 / \mathrm{F}$ & AML & 1.1 & 38.4 & Abnormal & - & 174 & 1 & 969 & 106 & 30 \\
\hline $3-2^{b}$ & & & 0.6 & 0.6 & Normal & - & 0 & 0 & 16 & 1 & 0 \\
\hline $4-1$ & $38 / \mathrm{M}$ & AML & 2.8 & 42.4 & Abnormal & + & 310 & 1 & 357 & 75 & 90 \\
\hline $4-2^{b}$ & & & 0.8 & 38.4 & Abnormal & - & 111 & 0 & 146 & 25 & $\mathrm{nc}$ \\
\hline 5 & $29 / \mathrm{F}$ & ALL & 1.7 & 98.3 & Abnormal & $\mathrm{nc}$ & $\mathrm{nc}$ & 0 & $\mathrm{nc}$ & 30 & 85 \\
\hline 6 & $72 / \mathrm{F}$ & AML & 0.3 & 13.7 & Normal & - & 15 & 0 & 71 & 7 & 80 \\
\hline 7 & $30 / \mathrm{M}$ & AML & 0.7 & 10.0 & Normal & - & 76 & 0 & 454 & 49 & 95 \\
\hline 8 & $64 / \mathrm{M}$ & AML & 0.8 & 8.1 & Normal & - & 1 & 1 & 29 & 2 & 44 \\
\hline 9 & $63 / \mathrm{M}$ & AML & 1.3 & 22.5 & Normal & - & 83 & 1 & 146 & 22 & 88 \\
\hline 10 & $31 / \mathrm{F}$ & AML & 1.1 & 18.6 & Normal & - & 46 & 0 & 113 & 15 & 60 \\
\hline 11 & $64 / \mathrm{M}$ & CLL & 0.5 & 12.6 & Normal & - & 3 & 0 & 5,321 & 440 & 100 \\
\hline
\end{tabular}

$D x$ Diagnosis, $T R$ total reticulocyte, $I R F$ immature reticulocyte fraction, UPP upper particle region, $n c$ not checked

a Flag displaying the message of "RET Abn Scattergram"

${ }^{\mathrm{b}}$ Data after therapeutic leukapheresis 
tosis (case nos. 7, 11), scattergram patterns were normal, and IRF values were not elevated. Interestingly, we found that the patients with an erroneously increased IRF had elevated UPP values and an abnormal scattergram. UPP values indicate erythroblast numbers and include some immature reticulocytes. Moreover, cases with an erroneously increased IRF had few normoblasts (zero to two normoblasts per $100 \mathrm{WBCs}$ ). All patients with an abnormal scattergram had a UPP value of more than 100 , while the UPP values of controls, which all had normal scattergram patterns, were less than 100 . WBC values depended on individual WBC counts. The flag message was less sensitive than abnormal reticulocyte scattergram in terms of the detection of a falsely increased IRF, because only three cases showed the flag (case nos. 1, 2, 4).

The dye used for reticulocyte analysis stains leukocytes and reticulocytes. Generally, leukocytes do not overlap with reticulocyte regions because of their high fluorescence. We hypothesized that insufficiently stained leukocytes of leukemic patients would have lower fluorescence than normal specimens and would be displayed in the UPP region and reticulocyte fraction. These low fluorescing leukocytes displayed in reticulocyte regions may be misidentified as immature reticulocytes, and thus, falsely elevated immature reticulocyte numbers.

In conclusion, leukocytes of leukemic patients that are insufficiently stained may have lower fluorescence, and thus, may be misidentified as immature reticulocytes and cause erroneously elevated IRF values in leukemia patients. The present study shows that in leukemia patients, when a reticulocyte scattergram pattern is abnormal and the UPP value is high, the IRF value may be erroneously high.

\section{References}

1. Riley RS, Ben-Ezra JM, Tidwell A, Romagnoli G (2002) Reticulocyte analysis by flow cytometry and other techniques. Hematol Oncol Clin North Am 16:373-420

2. Chang CC, Kass L (1997) Clinical significance of immature reticulocyte fraction determined by automated reticulocyte counting. Am J Clin Pathol 108:69-73

3. Noronha JF, De Souza CA, Vigorito AC, Aranha FJ, Zulli R, Miranda EC, Grotto HZ (2003) Immature reticulocytes as an early predictor of engraftment in autologous andallogeneic bone marrow transplantation. Clin Lab Haematol 25:47-54

4. Das R, Rawal A, Garewal G, Marwaha RK, Vohra H (2006) Automated reticulocyte response is a good predictor of bonemarrow recovery in pediatric malignancies. Pediatr Hematol Oncol 23:299-305

5. Choi JW, Pai SH (2003) Erythropoietic activities in acute leukemia and in malignant lymphoma with or without bone marrow involvement. Ann Clin Lab Sci 33:407-410

6. Villamor N, Kirsch A, Huhn D, Vives-Corrons JL, Serke S (1996) Interference of blood leucocytes in the measurements of immature red cells(reticulocytes) by two different semi-automated flow-cytometry technologies. Clin Lab Haematol 18:89-94 